Eur J Clin Chem Clin Biochem

$1995 ; 33: 417-424$

(c) 1995 Walter de Gruyter \& Co. Berlin - New York

\title{
Performance of a Direct; Immunoseparation Based LDL-Cholesterol Method Compared to Friedewald Calculation and a Polyvinyl Sulphate Precipitation Method
}

\author{
By Christa Cobbaert ${ }^{1}$. Ingrid Broodman ${ }^{1}$, G. Roel Swart ${ }^{2}$ and Nicoline Hoogerbrugge ${ }^{3}$ \\ 1 Department of Clinical Chemistry and Lipid Reference Laboratory \\ 2 Department of Internal Medicine II \\ 3 Department of Internal Medicine III and Lipid Clinic \\ University Hospital Dijkzigt, Rotterdam, The Netherlands
}

(Received September 23, 1994/May 2, 1995)

Summary: The analytical performance of a direct, immunoseparation based LDL-cholesterol method (Genzyme Corporation) was evaluated on an ELAN analyser (Merck), and compared with the performance of routinely used methods (LDL-cholesterol estimated by the Friedewald equation, and LDL-cholesterol obtained after polyvinyl sulphate precipitation). Within-day coefficients of variation (CVs) were 0.79 to $2.51 \%$ for immunoseparation based LDL-cholesterol; the between-day CVs varied between 2.62 and $3.89 \%$, i. e. within the recommended National Cholesterol Education Program (NCEP) goal of $<4 \%$. A method comparison study, according to the National Committee for Clinical Laboratory Standards (NCCLS) EP9-P guidelines, was performed using fasting normo- and hypertriacylglycerolaemic as well as cholestatic sera. In fresh normotriacylglycerolaemic sera immunoseparation based LDL-cholesterol (y) and Friedewald LDL-cholesterol $(\mathrm{x})$ values were identical as slope and intercept of the Passing \& Bablok regression equation were not significantly different from one and zero, respectively $(y=1.006 \mathrm{x}$ $-0.107 ; \mathrm{N}=45$ ). In contrast, immunoseparation based LDL-cholesterol (y) differed significantly from polyvinyl sulphate LDL-cholesterol $(x)$ results $(y=0.922 x+0.234 ; N=103)$. Freezing normotriacylglycerolaemic sera (three weeks, $-20^{\circ} \mathrm{C}$ ) resulted in a negative bias of $-5.8 \%$ for the immunoseparation based LDL-cholesterol method, and in a positive bias of $+5.3 \%$ for the polyvinyl sulphate method, compared to fresh specimens. Immunoseparated LDL-cholesterol was completely recovered up to at least $37.84 \mathrm{mmol} / \mathrm{l}$ serum triacylglycerols.

We conclude that the immunoseparation based LDL-cholesterol method is a practical, not technically demanding technique well applicable within routine clinical laboratories. The method shows a markedly improved analytical precision in comparison to current routine methods, and hence has potential to decrease total test imprecision. The immunoseparation based LDL-cholesterol method produces results identical to those obtained by Friedewald in healthy blood donors, and above all overcomes a major pitfall of the Friedewald equation enabling LDL-cholesterol measurements in hypertriacylglycerolaemic sera. Its acceptance should improve the reliability of LDL-cholesterol testing and improve clinical decision making.

\section{Introduction}

Serum LDL-cholesterol is an important risk factor for coronary heart disease $(1-2)$. Intervention trials have demonstrated that lowering (LDL)cholesterol either by diet or drug therapy decreased the incidence of coronary heart disease (3). From the Lipid Research Clinics Coro- nary Primary Prevention Trial it was concluded that each $1 \%$ reduction in serum LDL-cholesterol level decreased the incidence of coronary heart disease risk by $2 \%$. Because of the strong, positive link between LDL-cholesterol and coronary heart disease, both the European 
Atherosclerosis Society (4) as well as the Children and Adolescent Treatment Panel, and the Adult Treatment Panel (5-6) of the US National Cholesterol Education Program (NCEP) made LDL-cholesterol the focal point of classification of therapy, and stressed the importance of setting individual goal values for LDL-cholesterol. Consequently, LDL-cholesterol assays play a central role in the evaluation and management of hypercholesterolaemia.

Yet, measuring cholesterol in LDL is considerably more complicated than measuring total cholesterol, the latter not being influenced by the method of lipoprotein separation, or by cholesterol transfer between lipoproteins. For total cholesterol a Definitive Isotope Dilution/Mass Spectrometry Method and a Reference Abell-Kendall Method have been established to evaluate the accuracy of cholesterol analyses (7-8). For LDL-cholesterol no such formal standardization among lipoprotein laboratories exists.

In this study the analytical performance of a direct immunoseparation based LDL-cholesterol assay (9) was evaluated and compared with methods currently used in European routine clinical laboratories. The comparison methods were: LDL-cholesterol estimated according to Friedewald (10), and LDL-cholesterol obtained indirectly after polyvinyl sulphate precipitation of LDL and enzymatic determination of non-LDL-cholesterol in the supernatant. In the latter method LDL-cholesterol is calculated as the difference between total cholesterol, determined in neat serum, and cholesterol in the polyvinyl sulphate supernatant $(11-13)$. The aims of this evaluation study were fivefold: firstly, to apply the immunoseparation based LDL-cholesterol method to an automated clinical chemistry analyser so that the analytical variability is improved compared to current methods; secondly, to document the analytical variability for all three LDL-cholesterol methods, and to verify for which methods the recommended NCEP goal of $4 \%$ CV for LDL-cholesterol measurements could be reached (14); thirdly, to set up an LDL-cholesterol method comparison study in fresh fasting sera from selected healthy and diseased individuals - hypertriacylglycerolaemic and cholestatic patients - in order to document comparability with current routine methods and to check whether triacylglycerols and lipoprotein-X (Lp-X) affect the immunoseparation based LDL-cholesterol assay. Fourthly, to document the effect of freezing on immunoseparation based LDL-cholesterol. Finally, to evaluate indirectly the specificity of the immunoseparation step by checking the correlation between filtrate LDL-cholesterol and serum apolipoprotein $B$, between filtrate apolipoprotein $B$ and serum apolipoprotein $B$, and between filtrate lipoprotein(a) (Lp(a)) and serum $L p(a)$.

\section{Materials and Methods}

Patient sera

Fasting sera were obtained over a four month period by venipuncture from

1: apparently healthy normotriacylglycerolaemic blood donors ( $N=103: 58$ in the pilot study, 45 in the final study),

2: patients from the lipid clinic with known hypertriacylglycerolaemia or hospitalized patients with visually turbid sera due to elevated serum triacylglycerols $(\mathrm{N}=30)$, and

3: hospitalized patients with cholestasis $(N=12)$. In the cholestatic group five patients suffered from cirrhosis of the liver, five from acute hepatitis and two from obstructive jaundice.

Mean bilirubin, alkaline phosphatase ${ }^{1}$ ) and $\gamma$-glutamyltransferase ${ }^{\prime}$ ) levels were $124 \mu \mathrm{mol} / \mathrm{l}, 175 \mathrm{U} / \mathrm{l}$ and $200 \mathrm{U} / \mathrm{l}$ respectively. Minimum-maximum ranges were $21-371 \mu \mathrm{mol} / 1,78-430 \mathrm{U} / \mathrm{l}$ and 48-664 U/l, the respective corresponding upper reference limits being $14 \mu \mathrm{mol} / \mathrm{l}, 75 \mathrm{U} / \mathrm{l}$ and $35 \mathrm{U} / \mathrm{l}$.

Venous blood was drawn into vacuum tubes (Becton Dickenson). Plain tubes were left to clot for at least 30 minutes and centrifuged to obtain serum. For the method comparison study duplicate immunoseparation based LDL-cholesterol, polyvinyl sulphate LDL-cholesterol and total cholesterol analyses were performed the same day in fresh sera. Forty-five normotriacylglycerolaemic sera were aliquoted and stored frozen at $-20^{\circ} \mathrm{C}$ during three weeks. Duplicate immunoseparation based LDL-cholesterol and polyvinyl sulphate LDL-cholesterol determinations were repeated on frozen aliquots and compared to fresh LDL-cholesterol results. HDL-cholesterol, triacylglycerols, apolipoprotein B and Lp(a) measurements were all performed on frozen serum aliquots.

\section{Methods}

\section{LDL-cholesterol immunoseparation reagent}

The LDL-cholesterol Immunoseparation reagent (Cat. No. 246103, Genzyme Corporation, Cambridge, MA, USA) utilizes latex beads coated with immunoaffinity chromatography purified polyclonal goat antibodies to human apolipoproteins A-I and E. The antibodies are bound to separate populations of polystyrene latex beads, that are combined in a somewhat viscous reagent suspension. The antibody-coated beads selectively remove chylomicrons, HDL, IDL and VLDL lipoproteins from serum, while LDL and $\mathrm{Lp}$ (a) remain in the filtrate (Genzyme product information). A dual-chamber microcentrifuge filter unit consisting of a smaller inner tube equipped with filter, and a larger outer housing with cap, separates the HDL and VLDL-loaded beads from unbound LDL in the filtrate. The lot number of the reagent kits evaluated was D3316A.

Procedure used for the inmunoseparation based LDL-cholesterol determinations

Pipette $200 \mu \mathrm{l}$ of reagent into the dual-chamber microcentrifuge filter unit. Add $30 \mu \mathrm{l}$ of patient serum. Cap the device. and mix immediately by vortexing. Do not mix by inversion. Incubate for

1) Enzymes:

Cholesterol oxidase; cholesterol : oxygen oxidoreductase, EC 1.1.3.4

Glycero-phosphate oxidase; $L$-glycerol-3-phosphate : oxygen oxidoreductase, EC 1.1.3.21

$\gamma$-Glutamyltransferase; ( $\gamma$-glutamyl)-peptide : amino acid $\gamma$-glutamyl transferase, EC 2.3.2.2

Alkaline phosphatase; orthophosphoric monoester phosphohydrolase, EC 3.1.3.1 
10 minutes at room temperature. Centrifuge at room temperature for 5 minutes at $12000 \mathrm{~g}$. Remove and discard the inner tube. Assay the filtrate collected in the outer tube using a cholesterol assay. Correct the results for dilution of the sample with LDL reagent, by mcans of a batch-specific multiplication factor provided by Genzyme. The LDL immunoseparation reagent batch number used in this evaluation study was 3197B, the corresponding multiplication factor was 7.35 .

$L D L$ filtrates were analysed for cholesterol on an ELAN analyser (Merck, Darmstadt, Germany) using an application for low-level cholesterol measurements and an enzymatic cholesterol-oxidase')/ phenol-aminophenazone (CHOD-PAP) method (Monotest Cholesterol High Performance, Cat. No. 236691, Boehringer, Mannheim, Germany). Frozen human serum which was targeted with the cholesterol Abell-Kendall Reference Method (15) - quadruplicate analyses in four runs - was used as calibrator.

\section{LDL-cholesterol comparison methods}

Precipitation method: Polyvinyl sulphate LDL-cholesterol determinations were performed on supernatants after LDL precipitation from serum by polyvinyl sulphate, in the presence of EDTA and polyethylene glycol methyl ether (Cat. Nr. 726290, Boehringer, Mannheim, Germany), using Boehringer CHOD-PAP reagent (Monotest Cholesterol High Performance, Cat. No. 236691, Boehringer, Mannheim, Germany) on a semi-automated system. Polyvinyl sulphate LDL-cholesterol was calculated as the difference between total cholesterol, and cholesterol in the polyvinyl sulphate supernatant. Therefore, the polyvinyl sulphate method is an indirect LDL-cholesterol method. A Preciset ${ }^{\circledR}$ Cholesterol standard of 5.17 $\mathrm{mmol} / \mathrm{h}$ was used for calibration (Cat. Nr. 125512, Boehringer, Mannheim, Germany).

Estimated LDL-cholesterol: LDL-cholesterol was estimated as LDL-cholesterol = total cholesterol - HDL-cholesterol - (triacylglycerols/2.2), according to the original Friedewald formula (10). Cholesterol, LDL-cholesterol, HDL-cholesterol and triacylglycerol values are all expressed in mmol/l. In this study LDL-cholesterol could only be estimated in the blood donor group, because of documented limitations $(10,16)$.

Total and HDL-cholesterol determinations were carried out with a semi-automated procedure using Boehringer CHOD-PAP reagent (Monotest Cholesterol High Performance, Cat. No. 236691, Boehringer, Mannheim, Germany). HDL-cholesterol was measured in the supernatant after phosphotungstic acid/ $\mathrm{MgCl}_{2}$ precipitation of non-HDL particles (Cat. No. 14210, Merck, Darmstadt, Germany). Preciset ${ }^{\circledR}$ Cholesterol standards of 1.29 and $5.17 \mathrm{mmol} / 1$ were used for calibrating the HDL-cholesterol and total cholesterol assays, respectively (Cat. Nr. 125512, Boehringer, Mannheim, Germany).

The laboratory which performed the lipid analyses maintains total and HDL-cholesterol standardization through the Lipid Standardization Panel of the Centers for Disease Control (CDC) - National Heart Lung and Blood Institute. The laboratory is also a member of the Cholesterol Reference Method Laboratory Network (CRMLN) established by CDC (17).

Serum triacylglycerols without glycerol blanking were determined on a Chem 1 analyser (Technicon Inc., Tarrytown, New York, USA) using the UV method of Bucolo \& David (Technicon, Cat. No. T01-1656-53, method No. SA4-0324L90).

Apolipoprotein $B$ was determined on a Beckman Array with Beckman calibrator (Cat. Nr. 449370) and reagent (Cat. Nr. 449310) both in LDL filtrates and in neat serum, filtrates being diluted 6fold instead of 36 -fold.

Lipoprotein(a) ( $\mathrm{Lp}(\mathrm{a})$ ) was measured in neat serum and LDL filtrates using TintElize Lp(a) (Cat. Nr. 610220, Biopool, Umea, Sweden), filtrates being diluted 357-fold instead of 2601-fold. Results were expressed in $\mathrm{mg} / \mathrm{l} \mathrm{Lp}(\mathrm{a})$ mass.

\section{Quality control materials}

Genzyme human control sera were used to assess the within-day and day-to-day reproducibility for the immunoseparation based LDL-cholesterol method. The Genzyme controls investigated were direct LDL Cholesterol Desirable Level Control, and Risk Level Control (batch nr. D3298B with a target mean of $2.39 \mathrm{mmol} / \mathrm{l}$, and bath $\mathrm{nr}$. D3025A with a target mean of $5.29 \mathrm{mmol} / \mathrm{l}$ ).

Precinorm (Cat. No. 781827, Lot No. 180033, Boehringer Mannheim) and/or Seronorm ${ }^{\circledR}$ (Cat. No. 65, Lot No. 65, Nycomed) were used for daily quality control of cholesterol, HDL-cholesterol and polyvinyl sulphate LDL-cholesterol determinations, while for triacylglycerols Testpoint ${ }^{\mathrm{TM}} 1$ (Unassayed Chemistry Control 1, NORM, Technicon, Cat. No. T13-1070, Lot No. V09316) and Testpoint ${ }^{\mathrm{TM}} 2$ (Unassayed Chemistry Control 2, ABN, Technicon, Cat. No. T13-1071, Lot no. V093170) were used.

\section{Method comparison study}

The LDL-cholesterol comparison was performed essentially according to the NCCLS EP9-P protocol (18). The lipid distributions of the specimens tested in the final study were: $3.57-8.10 \mathrm{mmol} / \mathrm{l}$ for cholesterol, $1.58-5.98 \mathrm{mmol} / 1$ for polyvinyl sulphate LDLcholesterol, and $0.57-2.81 \mathrm{mmol} / \mathrm{l}$ for triacylglycerols in the blood donor subgroup; $3.65-15.47 \mathrm{mmol} / \mathrm{l}$ and $2.26-37.76 \mathrm{mmol} / \mathrm{l}$ respectively for cholesterol and triacylglycerols in the hypertriacylglycerolaemic group; and $1.94-11.88 \mathrm{mmol} / \mathrm{l}$ and $0.54-22.71$ $\mathrm{mmol} / \mathrm{l}$ respectively for cholesterol and triacylglycerols in the cholestatic group.

A comparison between all three LDL-cholesterol methods could only be performed in the normotriacylglycerolaemic patients $(\mathrm{N}=45)(10,16)$. Immunoseparation and polyvinyl sulphate derived LDL-cholesterol results were compared within the hypertriacylglycerolaemic and cholestatic patient groups.

\section{Triacylglycerol interference test}

A dilution series was prepared by mixing a high and a low triacylglycerol serum pool. The triacylglycerol range tested varied between 1.51 and $37.84 \mathrm{mmol} / 1$. Immunoseparation and polyvinyl sulphate derived LDL-cholesterol determinations were performed. LDL-cholesterol recovery was assessed by means of linear regression analysis (x: \% high pool, $\mathrm{y}$ : measured LDL-cholesterol).

\section{Statistical analysis}

Comparability of LDL-cholesterol methods was assessed by Bablok \& Passing regression analysis (19). A significance level of $\alpha=0.05$ was adopted.

\section{Results}

\section{Direct LDL-cholesterol application on ELAN}

The ELAN analyser is an open system enabling free programming of nearly all parameter settings. As approximately 7 -fold sample predilutions are made with the direct LDL-cholesterol immunoseparation reagent, a filtrate : reagent volume ratio of $25 \mu \mathrm{l}: 250 \mu \mathrm{l}$ was programmed to ensure adequate analytical sensitivity.

As the ELAN pipetting system was found to be matrix sensitive, a fresh frozen human serum cholesterol calibrator was used. Moreover, it was also necessary to di- 
lute the cholesterol calibrator like the LDL-cholesterol filtrates: before each run the calibrator was thawed and diluted 7.35-fold with physiological saline solution. Using this procedure matrix problems could be avoided. A mean absorbance of 0.4655 was measured at a 5.28 $\mathrm{mmol} / \mathrm{l}$ serum cholesterol concentration level, resulting in a sensitivity of $\Delta \mathrm{A}=0.088$ per $\mathrm{mmol} \mathrm{l}^{-1} \mathrm{LDL}$-cholesterol. The calibrator factor obtained was 11.232 , with a $\mathrm{CV}$ over the four month evaluation period of $1.50 \%$ $(\mathrm{N}=23)$.

\section{Within-run precision}

For the Desirable Level Control the CVs were 1.36, 2.51 and $2.38 \%$ over three consecutive days. For the Risk Level Control the CVs were $0.79,1.17$ and $1.44 \%$. The within-run mean values were $2.48,2.57$ and $2.43 \mathrm{mmol} / 1$ respectively for the desirable level, and 5.46, 5.49 and $5.35 \mathrm{mmol} / 1$ for the risk level. These within-run mean values represent twenty-one LDL immunoseparations analysed in a single run and repeated on three consecutive days.

\section{Between-day precision}

Mean analytical variability for respectively total cholesterol, triacylglycerols and HDL-cholesterol over the evaluation period was $0.86 \%, 2.09 \%$ and $4.17 \%$. Consequently, the calculated analytical variability of Friedewald LDL-cholesterol was $4.74 \%$. For polyvinyl sulphate LDL-cholesterol the between-day imprecision over a four month period $(\mathrm{N}=23)$ was $5.64 \%$ at 2.74 $\mathrm{mmol} / \mathrm{l}$. For immunoseparation based LDL-cholesterol the between-day $\mathrm{CV}$ over the same period $(\mathrm{N}=24)$ was $3.89 \%$ at $2.43 \mathrm{mmol} / \mathrm{l}$ and $2.64 \%$ at $5.37 \mathrm{mmol} / \mathrm{l}$, resulting in a mean CV of $3.27 \%$.

\section{Method comparison of immunoseparation based LDL-cholesterol with Friedewald LDL-cholesterol and polyvinyl sulphate LDL-cholesterol}

The Passing \& Bablok regression equations are presented in table 1. No outliers were present. In fresh normotriacylglycerolaemic sera slope and intercept for immunoseparation based LDL-cholesterol versus Friedewald LDL-cholesterol did not differ significantly from one and zero, respectively. In contrast, immunoseparation based LDL-cholesterol differed significantly from polyvinyl sulphate LDL-cholesterol. In hypertriacylglycerolaemic sera Friedewald LDL-cholesterol could not be estimated (10). A weak correlation existed between immunoseparation based LDL-cholesterol and polyvinyl sulphate LDL-cholesterol $(r=0.418)$, as well

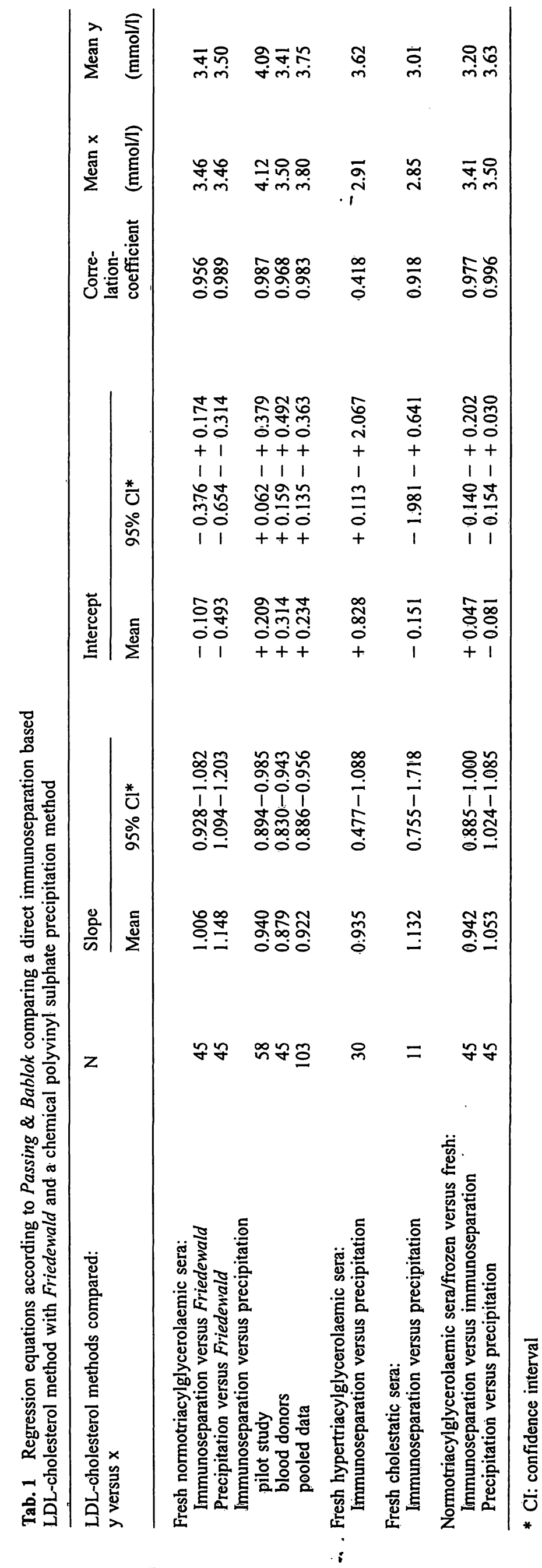


as a $20 \%$ mean difference (polyvinyl sulphate LDL-cholesterol: $2.91 \mathrm{mmol} / \mathrm{l}$; immunoseparation based LDLcholesterol: $3.62 \mathrm{mmol} / \mathrm{l})$, suggesting a different triacylglycerol effect on one or either LDL-cholesterol method. After immunoseparation none of the LDL filtrates looked turbid, while after polyvinyl sulphate precipitation nearly half of the supernatants remained turbid or non-homogeneous. Eliminating turbid polyvinyl sulphate supernatants from the method comparison improved the correlation $(r=0.902)$. In cholestatic patients Friedewald LDL-cholesterol could not be estimated because of the abnormal lipoprotein spectrum (16). The method comparison between immunoseparation based LDL-cholesterol and polyvinyl sulphate LDL-cholesterol showed somewhat scattered LDL-cholesterol results $(r=0.918)$.

Freezing samples reduced the immunoseparation based LDL-cholesterol mean from $3.41 \mathrm{mmol} / \mathrm{l}$ in fresh sera to $3.20 \mathrm{mmol} / 1$ in frozen sera (mean bias $=-6.2 \%$, slope $=0.942$ ). Freezing increased the polyvinyl sulphate LDL-cholesterol mean from $3.50 \mathrm{mmol} / \mathrm{l}$ in fresh samples to $3.63 \mathrm{mmol} / \mathrm{l}$ in frozen samples (mean bias $=+3.7 \%$, slope $=1.053$ ).

\section{Triacylglycerol interference}

Linear regression analysis on the triacylglycerol dilution series showed that the immunoseparation based LDLcholesterol method maintained linearity $(r=-0.998)$ and did not suffer from triacylglycerol interference up to a level of $37.84 \mathrm{mmol} / \mathrm{l}$, in contrast with the polyvinyl sulphate LDL-cholesterol method. Mean LDL-cholesterol recovery in the dilution series was $99.5 \%$ with the immunoseparation based method.

\section{Specificity of the LDL immunoseparation step}

A correlation coefficient of $\mathrm{r}=0.970$ was found in the blood donor subgroup when comparing filtrate LDLcholesterol with serum apolipoprotein $\mathrm{B}$, compared to $r=0.965$ for polyvinyl sulphate LDL-cholesterol versus serum apolipoprotein $\mathrm{B}$. The regression equation describing the relationship between the filtrate and serum apolipoprotein B was: $\mathrm{y}=-0,069+0.921 \mathrm{x}$ $(\mathrm{r}=0.983)$. The Passing \& Bablok equation describing the relationship between the filtrate and serum $\mathrm{Lp}(\mathrm{a})$ was: $y=-3.871+0.755 \times(r=0.981)$.

\section{Discussion}

LDL-cholesterol has been shown to be a major risk factor for coronary heart disease in clinical and observa- tional epidemiological studies $(1-3)$. Hence, determination of LDL-cholesterol is an essential part in the evaluation of dyslipidaemia, coronary heart disease risk classification and clinical management in individual patients. Reliable LDL-cholesterol measurements are particularly important because of the strong correlation between LDL-cholesterol and coronary heart disease risk, and because the expected effect of therapy may be relatively small (diet modification: 10 to $15 \%$ reduction; drug therapy: 15 to $>30 \%$ reduction). To detect these small, yet clinically important differences the total LDLcholesterol imprecision should be less than half of the expected response, i. e. $<5 \%$ (20). Current LDL-cholesterol methods have a total variability of about 8 to $11 \%$ $(20-21)$. Generally, efforts to decrease total variability involve reducing either analytical and/or biological variability.

Up to now, LDL-cholesterol was usually estimated by use of the Friedewald equation (10), which assumes that the amount of VLDL-cholesterol (in $\mathrm{mmol} / \mathrm{l}$ ) can be estimated by dividing the fasting serum triacylglycerol concentration by a factor of 2.2. Comparison of Friedewald LDL-cholesterol with ultracentrifugally obtained LDL-cholesterol yielded good correlations $(r=0.94$ to $0.99)$, depending on the patient population $(10,22)$. McNamara et al. (23) and Warnick et al. (24) documented that Friedewald LDL-cholesterol was adequate for risk classification of coronary heart disease patients since 84 to $86 \%$ and $90 \%$ of Friedewald LDL-cholesterol values were within $10 \%$ of ultracentrifugally determined LDL-cholesterol if serum triacylglycerol levels were $<2.3 \mathrm{mmol} / \mathrm{l}$. Yet, the Friedewald formula suffers from well-documented deficiencies: it can only be used in fasting sera; it is inaccurate in case of dysbetalipoproteinaemia, when triacylglycerol levels are greater than $4.52 \mathrm{mmol} / \mathrm{l}$, and in diseased patients who have altered concentrations and composition of lipoproteins (16). Also, the reliability of Friedewald LDL-cholesterol depends on the accurate measurement of total cholesterol, HDL-cholesterol and triacylglycerols. Poor precision in one or all of these measurements will contribute to the LDL-cholesterol analytical variability (20-21, 25-26). Ultimately, the biological variability in each of these three measurements will also contribute to the total variability of the LDL-cholesterol value (20-21, 25-26).

In an attempt to overcome the limitations of the Friedewald formula, indirect polyanion precipitation methods for LDL-cholesterol were developed $(11-13)$. The accuracy of these precipitation methods versus ultracentrifugation was reviewed recently $(22,27)$. From these studies it was concluded that Friedewald LDL-cholesterol results agreed better with ultracentrifugation results than those obtained with precipitation methods. More- 
over, the precipitation methods have shown to be less accurate and specific, and less reliable in the case of hypertriacylglycerolaemia because of co-precipitation of VLDL with LDL in a concentration-independent way (27). These findings illustrate the failure of the precipitation methods to overcome major pitfalls of the Friedewald formula.

Recently an immunoseparation based direct LDL-cholesterol assay was introduced. We found the method to be simple and easily applicable on an automated clinical chemistry analyser. Within-run and between-day imprecision data for the immunoseparation based LDLcholesterol method illustrate that the analytical $\mathrm{CV}$ was improved substantially compared to current routine LDL-cholesterol methods, and remained below the NCEP recommended goal of $4 \%$ (14). The analytical imprecision of the immunoseparation based LDL-cholesterol method no longer depends upon the cumulative analytical variability present in the measurements of triacylglycerols, HDL-cholesterol and total cholesterol (for estimation of Friedewald LDL-cholesterol), or total and non-LDL-cholesterol (for calculation of polyvinyl sulphate LDL-cholesterol). For the same reason the biological variability should also improve. Consequently, the immunoseparation based method has the potential to reduce total test imprecision and to improve clinical decision making. Further studies to document total test variability and its impact on coronary heart disease risk classification are warranted.

The method comparison study (tab. 1) showed that there was excellent agreement between immunoseparation based LDL-cholesterol and Friedewald LDL-cholesterol in normotriacylglycerolaemic sera. Good specificity of the immunoseparation based LDL-cholesterol method was to be expected since the reagent contains highaffinity polyclonal antibodies to human apolipoproteins A-I and E, separating LDL particles on the basis of their characteristic apolipoprotein composition. Chylomicrons contain both apolipoproteins A-I and E, VLDL and LDL contain apolipoprotein E, HDL contain apolipoprotein A-I and sometimes apolipoprotein E. Vesicular lipoproteins like Lp-X also contain apolipoprotein $\mathrm{E}$ $(28,29)$. If present, all these particles should be expected to bind to the latex-coated antibodies. In contrast, LDL and $\mathrm{Lp}(\mathrm{a})$ which do not transport apolipoproteins A-I and E, are collected in the filtrate, and reported as 'LDL-cholesterol'. In this study we found that $\mathrm{Lp}(\mathrm{a})$ was not completely recovered in the LDL filtrate, the mean recovery being only $75 \%$ (tab. 1 ). The reason for this finding is unclear, however matrix effects related to the $L p(a)$ assay may have caused the difference. $L i$ et al. (30) documented that the average overestimation of Friedewald LDL-cholesterol was $4.1,8.5$ and $21.4 \%$ at
$\mathrm{Lp}$ (a) concentrations of $<300 \mathrm{mg} / \mathrm{l}, 301-600 \mathrm{mg} / \mathrm{l}$ and $>600 \mathrm{mg} / \mathrm{l}$ respectively. As Friedewald LDL-cholesterol and polyvinyl sulphate LDL-cholesterol results include $\mathrm{Lp}(\mathrm{a})$-cholesterol just like the immunoseparation based LDL-cholesterol method, the method comparison data produced by either method reflect the same lipoprotein classes. Although measurement of Friedewald, polyvinyl sulphate and immunoseparation based LDLcholesterol may be a sensitive and useful gauge of the sum of these two atherogens, the separation of $L p(a)$ cholesterol from LDL-cholesterol should be made in future LDL-cholesterol assays as there is evidence that LDL and Lp(a) have different prognostic significance (31).

While the polyvinyl sulphate method and the Friedewald equation cannot be used in case of even moderate hypertriacylglycerolaemia $(>4.52 \mathrm{mmol} / \mathrm{l})$, no triacylglycerol interference could be documented in the immunoseparation based LDL-cholesterol method up to $37.84 \mathrm{mmol} / \mathrm{l}$, enabling valid LDL-cholesterol determinations in almost every hypertriacylglycerolaemic patient, and potentially in non-fasting subjects. The observation that LDL filtrates from the triacylglycerol dilution series were transparent and visually clear after immunoextraction corroborates the absence of triacylglycerol interference.

When comparing immunoseparation based LDL-cholesterol versus polyvinyl sulphate LDL-cholesterol slope and intercept differed significantly from one and zero, respectively, even in normotriacylglycerolaemic sera. Fresh sera should be analysed in the immunoseparation based LDL-cholesterol method since freezing (three weeks, $-20^{\circ} \mathrm{C}$ ) introduced a significant bias of $-5.8 \%$ versus fresh sera.

In cholestasis the presence of $\mathrm{Lp}-\mathrm{X}$ is likely (16). Although both immunoseparation based LDL-cholesterol and polyvinyl sulphate LDL-cholesterol do not measure Lp-X ((22), manufacturers' product information), correlated but scattered LDL-cholesterol results were obtained. We hypothesize that this is probably caused by different bilirubin interference due to different sample : reagent ratios in the enzymatic cholesterol assays used.

Recently, Mc Namara et al. have evaluated 'accuracy' of the immunoseparation based LDL-cholesterol method versus the Lipid Research Clinics Beta-Quantification method (32). The Beta-Quantification method involves both an ultracentrifugation and a precipitation step. Briefly, VLDL is removed from neat serum by ultracentrifugation at serum density, and LDL is precipitated from the infranatant by dextran sulphate- $\mathrm{MgCl}_{2}$. VLDL ultracentrifugation infranatant and LDL precipitation supernatant are measured for cholesterol concentration. 
LDL-cholesterol is determined by the difference between the VLDL infranatant cholesterol value and the HDL supernatant cholesterol value (33). Besides its complexity, the Beta-Quantification method has some major disadvantages: firstly, the use of a precipitation method to determine HDL is subject to inaccuracies; secondly, LDL values are not measured directly, but are determined by the difference between VLDL infranatant and HDL supernatant. Therefore, the reported LDL value includes other lipoprotein species such as $L p(a)$ and IDL. Yet, as large population studies over the last 40 years have reported LDL-cholesterol concentrations traceable to the Beta-Quantification method, the latter will presumably become the future Reference Method.

In summary, the immunoseparation based direct LDLcholesterol method overcomes major drawbacks of cur-

\section{References}

1. Kannel WB, Castelli WP, Gordon T, McNamara PM. Serum cholesterol, lipoproteins and risk of coronary heart diseases. The Framingham study. Ann Intern Med 1971; 71:1-12.

2. Gordon T, Kannel WB, Castelli WP, Dawber TR. Lipoproteins, cardiovascular disease and death: the Framingham study. Arch Intern Med 1981; 141:1128-31.

3. Lipid Research Clinics Program. The Lipid Research Clinics Coronary Primary Prevention Trial results. II: The Relationship of reduction in incidence of coronary heart disease to cholesterol lowering. J Am Med Ass 1984; 251: 365-74.

4. Recommendations of the European Atherosclerosis Society prepared by the International Task Force for Prevention of Coronary Heart Disease. Prevention of coronary heart diseases: scientific background and new clinical guidelines. Nutr Metab Cardiovasc Dis 1992; 2:113-56.

5. National Cholesterol Education Program. Report of the Expert Panel on Blood Cholesterol Levels in Children and Adolescents. NIH Publication 91-2732. Bethesda, MD: National Institutes of Health, National Heart, Lung and Blood Institute, 1991:1-119.

6. Expert Panel on Detection, Evaluation, and Treatment of High Blood Cholesterol in Adults. Summary of the Second Report of the National Cholesterol Education Program (NCEP) Expert Panel on detection, evaluation, and treatment of high blood cholesterol in adults. Adult Treatment Panel II. J Am Med Ass 1993; 269:3015-23.

7. Cohen A, Hertz HS, Mandel J, Paule RC, Schaffer R, Sniegoski LJ, et al. Total serum cholesterol by isotope dilution/ mass spectrometry: a candidate definitive method. Clin Chem 1988; 34:193-201.

8. Duncan IW, Mather A, Cooper GR. The procedure for the proposed cholesterol reference method. Atlanta, GA: Centers for Disease Control, 1982.

9. Harina BM, Ollington JF. Direct method for LDL-C measurement using the Genzyme immunoseparation reagent [abstract]. Clin Chem 1992; 38:1065.

10. Friedewald WT, Levy RI, Fredrickson DS. Estimation of the concentration of low-density lipoprotein cholesterol in plasma, without use of the preparative ultracentrifuge. Clin Chem $1972 ; 18: 499-502$.

11. Demacker PN, Hijmans AG, Brenninkmeijer BJ, Jansen AP, van 't Laar A. Five methods for determining low-density lipoprotein cholesterol compared. Clin Chem 1984; 30:1797-800. rent routine LDL-cholesterol methods. Firstly, the decreased analytical variability of the direct LDL-cholesterol method will reduce total test variability, allowing clinicians to accurately assess coronary heart disease risk with only one or two blood specimens, and allow a more reliable assessment of response to diet and drug therapy. Secondly, the direct LDL-cholesterol method will also improve biological variability. Thirdly, the direct LDL-cholesterol method produces results identical to Friedewald, including both LDL- and Lp(a)-cholesterol. Finally, reliable LDL-cholesterol measurements can be performed in severe hypertriacylglycerolaemic samples, and be made with non-fasting specimens. The high price/test should limit its use to well-considered requests. We conclude that the immunoseparation based direct LDL-cholesterol assay is a major improvement compared to current routine LDL-cholesterol methods.

12. Hoffmann GE, Hieflinger R, Weiss L, Poppe W. Five methods for measuring low-density cholesterol concentration in serum compared. Clin Chem 1985; 31:1729-30.

13. Kerscher L, Schiefer S, Draeger B, Maier J, Ziegenhorn J. Precipitation methods for the determination of LDL-cholesterol. Clin Biochem 1985; 18:118-25.

14. Bachorik PS. Measurement of triglycerides and lipoproteins. The fats of life. Lipids and Lipoprotein Division, AACC. Washington, DC: American Association for Clinical Chemistry Publications, 1992:9-10.

15. Cooper GR, Myers GL, Henderson LO. Establishment of reference methods for lipids, lipoproteins and apolipoproteins. Eur J Clin Chem Clin Biochem 1991; 29:269-75.

16. Matas C, Cabré M, La Ville A, Prats E, Joven J, Turner PR, et al. Limitations of the Friedewald formula for estimating lowdensity lipoprotein cholesterol in alcoholics with liver disease. Clin Chem 1994; 40:404-6.

17. Wiebe DA, Westgard JO. Cholesterol - a model system to relate medical needs with analytical performance. Clin Chem 1993; 39:1504-13.

18. NCCLS Proposed Guideline EP9-P. User comparison of quantitative clinical laboratory methods using patient samples. National Committee for Clinical Laboratory Standards, volume 6, number 1 .

19. Passing $\mathrm{H}$, Bablok W. A new biomedical produre for testing the equality of measurements from two different analytical methods. J Clin Chem Clin Biochem 1983; 21:709-20.

20. Schectman G, Sasse E. Variability of lipid measurements: relevance for the clinician. Clin Chem 1993; 39:1495-503.

21. Cooper GR, Myers GL, Smith J, Schlant RC. Blood lipid measurements. Variations and practical utility. J Am Med Ass 1992; 267:1652-60.

22. Rifai N, Warnick R, McNamara JR, Belcher JD, Grinstead GF, Frantz D Jr. Measurement of low-density-lipoprotein cholesterol in serum: a status report. Clin Chem 1992; 38:150-62.

23. McNamara J, Cohn JS, Wilson PWF, Schaefer EJ. Calculated values for low-density lipoprotein cholesterol in the assessment of lipid abnormalities and coronary disease risk. Clin Chem 1990; 36:36-42.

24. Warnick R, Knopp RH, Fitzpatrick V, Branson L. Estimating low-density lipoprotein cholesterol by the Friedewald equation is adequate for classifying patients on the basis of nationally recommended cutpoints. Clin Chem 1990; 36:15-9. 
25. Bookstein L, Gidding SS, Donovan M, Smith FA. Day-to-day variability of serum cholesterol, triglyceride, and high-density lipoprotein cholesterol levels. Impäct on the assessment of risk according to the National Cholesterol Education Program Guidelines. Arch Intern Med 1990; 150:1653-7.

26. Marcovina SM, Gaur VP, Albers JJ. Biological variability of cholesterol, triglyceride, low- and high-density lipoprotein cholesterol, lipoprotein(a), and apolipoproteins A-I and B. Clin Chem 1994; 40:574-8.

27. Siekmeier R, März W, Groß W. Insufficient accuracy and precision of polyanion precipitation methods for quantifying low-density lipoproteins. Clin Chem 1990; 36:2109-13.

28. Williams KJ, Tall AR, Tabas I, Blum C. Recognition of vesicular lipoproteins by the apolipoprotein B, E receptor of cultured fibroblasts. J Lipid Res 1986; 27:892-900.

29. Hickman PE, Dwyer KP, Masarei JR. Pseudohyponatremia, hypercholesterolaemia, and primary biliary cirrhosis. J Clin Pathol 1989; 42:167-71.

30. Li KM, Wilcken DEL, Dudman NPB. Effect of serum lipoprotein(a) on estimation of low-density lipoprotein cholesterol by the Friedewald formula. Clin Chem 1994; 40:571-3.
31. Armstrong VW, Cremer P, Eberle E, Manke A, Schulze F, Wieland $\mathrm{H}$, et al. The association between $\mathrm{Lp}(\mathrm{a})$ concentration and angiographically assessed coronary atherosclerosis. Dependence on serum LDL levels. Atherosclerosis 1986; 62:249-57.

32. McNamara JR, Cole TG, Contois JH, Ferguson CA, Ordovas JM, Schaefer EJ. Immunoseparation method for measuring low-density lipoprotein cholesterol directly from serum evaluated. Clin Chem 1995; 41:232-40.

33. Manual of laboratory operations, lipid research clinics program, lipid and lipoprotein analysis. Whashington DC: NIH, US Department of Health and Human Services, 1982.

C. Cobbaert

Centraal Klinisch Chemisch Laboratorium Lipiden Referentie Laboratorium Academisch Ziekenhuis Dijkzigt

Dr. Molewaterplein 40

NL-3015 GD Rotterdam

The Netherlands 